\title{
Carbon C 14 Lenvatinib Mesylate
}

National Cancer Institute

\section{Source}

National Cancer Institute. Carbon C 14 Lenvatinib Mesylate. NCI Thesaurus. Code C124797.

A radioconjugate composed of the orally bioavailable mesylate salt form of lenvatinib, a receptor tyrosine kinase (RTK) inhibitor, labeled with the radioisotope carbon C 14, with potential use for evaluating the pharmacokinetic profile of lenvatinib. Upon administration of carbon C14 lenvatinib mesylate, lenvatinib targets and binds strongly to multiple RTKs, including vascular endothelial growth factor receptor 1 (VEGFR1;FLT1), VEGFR2 (KDR), VEGFR3 (FLT4), fibroblast growth factor receptor 1 (FGFR1), FGFR2, FGFR3, and FGFR4, platelet derived growth factor receptor alpha (PDGFRa), KIT, and RET. This inhibits growth of tumor cells that overexpress these RT Ks. Labeling of lenvatinib with the radioactive tracer carbon C 14 permits the evaluation of lenvatinib's pharmacokinetic profile, including its absorption, distribution, metabolism, and excretion (ADME). 